\title{
ENSEÑANZA DEL INGLÉS EN EDUCACIÓN RURAL: ERCILLA- COLLIPULLI, CHILE
}

\author{
Gabriel Eduardo Anríquez Ponce \\ Universidad de la Frontera - gabrielanriquez@gmail.com
}

\begin{abstract}
Resumen: Esta investigación tiene por objeto describir las prácticas profesionales que realizan los docentes en la Red de Inglés Ercilla- Collipulli Rural English Net, pertenecientes a escuelas municipales y particulares subvencionadas de las comunas de Ercilla y Collipulli, en Chile, y determinar los factores que influyen en el éxito o fracaso del uso del recurso educativo denominado It's my turn. Para dar cumplimiento al objetivo fueron entrevistados un total de diecinueve profesores. Los resultados obtenidos dan cuenta que el trabajo realizado en este espacio constituye una práctica consolidada, lo que les permitió establecer las fortalezas y debilidades del grupo. Entre sus fortalezas se encuentran el intercambio de experiencias, la transposición al aula de lo trabajado en la Red, la mejora en la disposición de los docentes para enfrentar la enseñanza del idioma inglés, la responsabilidad frente a los compromisos, la valoración por las opiniones divergentes, la sistematización de sus prácticas y el compromiso del coordinador de la red en todas las actividades realizadas, mientras que entre sus debilidades reconocen la falta de acompañamiento constante por parte del PIAP, la distancia entre las escuelas participantes y el lugar de reunión y la poca formación en el idioma inglés por parte de los profesores que integran la Red.

En relación a los factores que influyen en el éxito o fracaso del uso del recurso educativo denominado It's my turn se desprende que es esencial para el éxito la disposición para emplear el material y la confianza en sus capacidades profesionales.
\end{abstract}

Palabras clave: Enseñanza idioma Inglés, Educación Rural, Aula Multigrado, Aprendizaje colaborativo, Trabajo en redes.

\section{INTRODUCCIÓN}

De acuerdo a información obtenida del Programa Inglés Abre Puertas (PIAP), la región de la Araucanía, Chile, posee 561 docentes que imparten inglés, de los cuales 224 (40\%) no posee formación para hacerlo. Con la finalidad de contribuir a la enseñanza de este idioma el PIAP ha puesto a disposición de los docentes distintas iniciativas: examen de posicionamiento, cursos de nivelación, charlas, seminarios, encuentros, recursos educativos, Redes de docentes de inglés, entre otros.

Una de las iniciativas fueron las redes de inglés, siendo una de ellas la Red Ercilla- Collipulli Rural English Net, que inicia sus funciones el año 2011. Así como esta red existen otras con similares características en la región y el país, cada una de las cuales elaboran y ejecutan un plan de acción. De acuerdo a la revisión de la literatura se da cuenta de la ausencia de investigaciones que aborden las prácticas docentes abordadas en las redes de inglés nacidas al alero del PIAP. 
El programa PIAP provee recursos educativos, uno de ellos es It's my turn, distribuido desde 2010 en los distintos establecimientos educacionales que imparten Inglés en las escuelas rurales y del que tampoco existen evidencias que den cuenta de sus resultados ni de la percepción que los docentes tienen de él.

Desde la mirada de Weiss (1998) es necesario evaluar los programas en función de las metas que se propuso alcanzar, a fin de contribuir a la toma de decisiones y a la mejora de la programación futura. En este sentido esta investigación busca describir las prácticas profesionales que realizan los docentes en la Red de Inglés ErcillaCollipulli Rural English Net, pertenecientes a escuelas municipales y particulares subvencionadas de las comunas de Ercilla y Collipulli, en Chile, y determinar los factores que influyen en el éxito o fracaso del uso del recurso educativo denominado It's my turn.

\section{PERSPECTIVA TEÓRICA}

\subsection{Enseñanza del Inglés como idioma extranjero}

La enseñanza del Inglés como idioma extranjero debe considerar como punto de partida el conocimiento del estudiante acerca del mundo que lo rodea $y$, a partir de aquí, establecer una forma de enseñar nuevos aprendizajes, procurando un equilibrio entre la seguridad que da basarse en sus conocimientos previos y el interés proporcionado por la presentación creativa de nuevos aprendizajes (Criado, 2008).

La enseñanza del inglés en las escuelas busca contribuir a que los estudiantes adquieran y desarrollen competencias que les permitan enfrentarse de mejor forma a esta lengua a partir de las características de los educandos. Ahora, considerando que el PIAP está enfocado en atender principalmente a estudiantes de Quinto y Sexto año Básico, es preciso considerar las particularidades de los niños de 10 y 11 años, quienes se caracterizan por su energía y vitalidad, por la necesidad de movimiento y del uso espontáneo del cuerpo, lo cual puede ser utilizado en el aprendizaje de inglés a través de actividades de mímica, de acción y de comunicación (Criado, 2008), para lo que el docente puede emplear uno de los recursos que forma parte de It's my turn.

Para la enseñanza del idioma inglés como idioma extranjero resulta conveniente que el profesorado realice acciones que apoyen la 
comunicación verbal, reforzando así palabras y estructuras. Con esto se busca que el estudiante, por imitación, replique estos gestos y en la medida que vaya mejorando su expresión oral pueda prescindir de este tipo de apoyo. No obstante, y dado que los períodos de atención de los niños y niñas de estas edades son cortos, es conveniente que haya un equilibrio entre las actividades que implican movimiento y otras que requieran concentración.

Por otro lado, el estudiante de estas edades es por lo general imaginativo, divertido, entusiasta y creativo por lo que resulta sencillo incluir a los niños y niñas en actividades lúdicas. En este sentido es muy útil el empleo de dinámicas como las propuestas en el recurso It's my turn, entre las que se encuentran canciones, poemas y diálogos.

\subsection{Recurso educativo It's my turn}

El año 2010 el Programa Inglés Abre Puertas (PIAP) presentó el recurso educativo It's my turn con el propósito de apoyar la labor del docente no especialista y de satisfacer las necesidades de enseñanzaaprendizaje del idioma inglés en los niveles de $5^{\circ}$ y $6^{\circ}$ año de enseñanza básica en establecimientos rurales de Chile, obligatorio desde el año 2010 según el DS n40 de 1996.

El objetivo de este recurso educativo es que los estudiantes aprendan inglés junto a sus profesores, convirtiéndose estos últimos en facilitadores del proceso mediante un set de clases pregrabadas en formato DVD, con lecciones y actividades diseñadas para cada clase, cubriendo así todo el año escolar.

Entre las características de este recurso educativo destaca que sus personajes viven en el campo y asisten a un aula multigrado, contexto similar a las escuelas donde se aplica, lo que de cierta forma hace sentir al estudiante más cercano a los protagonistas del software. Para apoyar la labor de facilitador del contenido, el docente cuenta con las Guías Didácticas que contienen todas las respuestas a los ejercicios de los cuadernillos del estudiante, además de un Cuaderno de Evaluación y un Cuaderno de Planificación. Cada uno de los niveles de este recurso cuenta con cinco unidades, dentro de las cuales hay de 7 a 9 lecciones, siendo la última unidad de cada nivel una revisión de los contenidos trabajados durante el año. 
Este recurso emplea las TICs en la enseñanza del inglés y desarrolla la competencia de autoaprendizaje, entendido como aquel que fomenta en los estudiantes la capacidad de llevar a cabo opciones adecuadas que los haga más conscientes de sus actividades de estudio y menos dependiente del profesor y de los materiales didácticos (Montalto, 1997).

\subsection{Redes de Docentes de Inglés}

Dentro de las diversas iniciativas que promueve el PIAP se encuentran las Redes de Docentes de Inglés, comunidades de aprendizaje conformadas por docentes de inglés de los distintos establecimientos de escuelas multigrado a lo largo de todo Chile.

Estas Redes surgen de la necesidad de reflexionar y colaborar entre pares con la finalidad de adaptarse a las nuevas demandas que plantea la enseñanza y aprendizaje del inglés en el sistema escolar chileno. Es así que los docentes que las constituyen se reúnen periódicamente a discutir temas y problemáticas que surgen de la enseñanza del inglés en el aula, diseñando recursos y estrategias para mejorar y potenciar sus prácticas docentes.

Los docentes de las redes rurales funcionan en el marco del aprendizaje colaborativo que está centrado en la interacción de sus miembros y en lo que cada uno puede aportar al grupo. Como indican Johnson y Johnson (2000) el aprendizaje colaborativo es un sistema de interacciones que organiza e induce la influencia recíproca entre los integrantes de un equipo y que se desarrolla a través de un proceso gradual en el que cada miembro se siente comprometido con el otro, generando una interdependencia positiva que no implique competencia.

Las redes de docentes de inglés buscan que la autoridad sea compartida y se acepte la responsabilidad y las opiniones de otros miembros, principios del aprendizaje colaborativo, lo que para Gros (2000) sólo puede conseguirse si el trabajo del grupo se basa en la colaboración. En esta modalidad de aprendizaje es el grupo el que toma las decisiones de cómo realizar la tarea, de los procedimientos que se deben adoptar, de cómo se realiza la división del trabajo y de qué tareas se deben realizar para conseguir alcanzar los objetivos (Chiarani \& Pianucci, 2003). 


\subsection{Trabajo en redes y aprendizaje colaborativo}

Una red de docentes es entendida como un grupo de profesionales que se reúnen voluntariamente para hablar y crear acuerdos teóricos y prácticos (Birchak et. al., 1998), por lo que la red requiere un sentido de comunidad entre los docentes, destinado a apoyar el desarrollo profesional y a transformar la enseñanza a través del diálogo y la reflexión.

El trabajo en red sucede en el marco del aprendizaje colaborativo inmerso en la teoría del constructivismo social (Gosden, 1994) que centra su atención en el proceso de construcción del conocimiento a través del aprendizaje que resulta de la interacción con un grupo, mediante tareas realizadas en cooperación con otros. De esta forma, el aprendizaje colaborativo entonces define el cómo aprendemos (socialmente) y dónde aprendemos (en red).

Algunos de los autores que definieron el aprendizaje colaborativo y establecieron los principales elementos de éste fueron los psicólogos Johnson y Johnson (1986) y Slavin (1989). Para estos autores los elementos que debían estar presentes en una situación de aprendizaje colaborativo eran: a) Interdependencia positiva, donde los miembros de un grupo persiguen un objetivo común y comparten recursos e información; b) Promoción a la interacción, donde los miembros de un grupo se ayudan unos a otros para trabajar eficiente y efectivamente mediante la contribución individual de cada sujeto; c) Responsabilidad individual donde cada uno de los miembros del grupo es responsable por su aporte individual y por la manera que ese aporte contribuye al aprendizaje de todos; d) Habilidades y destrezas de trabajo grupales donde cada uno de los miembros debe comunicarse, apoyar a otros, y resolver conflictos con otro miembro constructivamente; y e) Interacción positiva dado que cada uno de los miembros debe mantener una buena relación de cooperación con los otros y estar dispuesto a dar y recibir comentarios y críticas constructivas sobre sus contribuciones. Por lo tanto se puede establecer que las redes de inglés para docentes no especialistas en este idioma pueden contribuir positivamente a crear colaborativamente una amplia gama de estrategias, actividades y técnicas en la enseñanza-aprendizaje. 


\section{METODOLOGÍA}

La investigación adscribe al paradigma constructivista y el diseño corresponde al estudio de caso, definido por Stake (1998) como "el estudio de la particularidad y complejidad de un caso singular, para llegar a comprender su actividad en circunstancias importantes" (p. 11).

\subsection{El caso: Ercilla- Collipulli Rural English Net, Región de La Araucanía, Chile.}

La Red está conformada por docentes de 11 escuelas rurales multigrado de la comuna de Ercilla y 8 escuelas de la comuna de Collipulli. Estas comunas pertenecen a la provincia de Malleco, Región de La Araucanía. Ercilla, por una parte cuenta con 7.733 habitantes y un $46 \%$ de población perteneciente al pueblo mapuche (BCN, 2015; e INE, 2017). El 44.3\% de su población se encuentra en situación de pobreza, superando el promedio regional (27.9\%) y el promedio nacional (14.4\%), según la Encuesta de Caracterización Social (BCN, 2015). Por otra parte, Collipulli posee 24.598 habitantes y un $26.43 \%$ de población perteneciente al pueblo mapuche (Municipalidad de Collipulli, 2018). El 32.20\% de su población se encuentra en situación de pobreza, superando el promedio nacional (14.4\%), según la Encuesta de Caracterización Social (BCN, 2015).

Esta red de inglés nace el año 2010, siendo reconocida por el Ministerio de Educación de Chile mediante Resolución Exenta $N^{\circ} 1305$ del 27 de abril de 2011. Los docentes que la componen se reúnen una vez al mes en dependencias del Liceo de Ercilla o del Internado de Collipulli, en reuniones de tres horas cronológicas donde revisan documentos y material didáctico proporcionados por el PIAP, estrategias de enseñanza, información de perfeccionamiento docente en idioma inglés, entre otros temas.

Esta red postuló y ganó en 2012 y 2013 el proyecto English Week, del Ministerio de Educación, destinado a realizar actividades que propicien instancias para que los estudiantes puedan hacer uso del idioma inglés y demostrar el progreso alcanzado variadas situaciones, promoviendo el trabajo colaborativo, interacción y aprendizaje entre pares tanto de docentes como estudiantes.

\subsection{Participantes}


Esta investigación contó con la colaboración de 19 docentes de Enseñanza Básica que imparten la asignatura de Inglés en escuelas rurales multigrigrado, de administración municipal o particular subvencionada de las comunas de Ercilla y Collipulli.

Las edades de los participantes, siete hombres y doce mujeres, van desde veintisiete hasta los cincuenta y seis años, mientras que los años de experiencia docente oscilan entre los cuatro y los treinta años.

\subsection{Técnicas e instrumentos de recolección de datos.}

Para la recolección de datos se empleó la técnica de entrevista semiestructurada. Se entrevistó a docentes que participan de la red mediante un formato de entrevista que permite realizar preguntas abiertas, permitiendo que el informante pueda expresar sus opiniones e ir elaborando respuestas que permitan profundizar sus ideas a medida que avanza la entrevista. La entrevista fue diseñada en función de dos tópicos: prácticas profesionales que realizan los docentes en la Red de Inglés Ercilla- Collipulli Rural English Net y los factores que influyen en el éxito o fracaso del uso del recurso educativo denominado It's my turn.

\subsection{Procedimiento y análisis de datos}

Para la recogida de información y análisis de los datos se siguió la propuesta de Krueger (1998). Así, primero se informó en una reunión de la Red acerca de los alcances de la investigación a los docentes que la conforman y luego se les invitó a participar en entrevistas semiestructuradas, cuyas interrogantes estaban orientadas a dar respuestas a la pregunta y objetivos de esta investigación. El segundo paso consistió en coordinar con los docentes los días y horarios de las entrevistas, las que fueron escritas en archivos de Microsoft Office Word. Por último los resultados obtenidos de las entrevistas realizadas fueron compartidos con los participantes.

\section{RESULTADOS}

Los resultados de esta investigación fueron separados en dos grandes tópicos: prácticas profesionales que realizan los docentes en la Red de Inglés Ercilla- Collipulli Rural English Net y factores que influyen en el éxito o fracaso del uso del recurso educativo denominado It's my turn. 
En relación al primer tópico, los docentes valoran el trabajo realizado en la Red dado que les permite compartir experiencias e intercambiar recursos relativos a la enseñanza del idioma inglés en el contexto rural multigrado, valorando en especial las dependencias del Internado de la comuna de Collipulli donde se realizan las reuniones, dado que consideran que este lugar les provee la comodidad para desarrollar sus actividades.

Con el fin de realizar un trabajo en un ambiente colaborativo y que apunte a un bien común, los docentes sostienen que acordaron establecer normas de trabajo para la Red de Ingles, entre las que se encuentran: opinar libremente, ser tolerante con las opiniones de los demás docentes, colaborar activamente con el trabajo de la red, compartir experiencias que constituyan un aporte a las prácticas pedagógicas de los demás profesores, y sobre todo, promover el bien común.

Al referirse a las actividades realizadas en la Red, los docentes participantes señalan que el trabajo realizado en este espacio constituye una práctica consolidada, lo que les permitió establecer las fortalezas y debilidades del grupo. Entre las fortalezas destacan el intercambio de experiencias, la transposición al aula de lo trabajado en la Red, la mejora en la disposición de los docentes para enfrentar la enseñanza del idioma inglés, la responsabilidad frente a los compromisos, la valoración por las opiniones divergentes, la sistematización de sus prácticas y el compromiso del coordinador de la red en todas las actividades realizadas. Entre las debilidades en tanto, reconocen la falta de acompañamiento constante por parte del PIAP, la distancia entre las escuelas participantes y el lugar de reunión y la poca formación en el idioma inglés por parte de los profesores que integran la Red.

En relación al segundo tópico, destaca que en un principio los docentes no tenían la disposición de implementar el recurso It's my turn, dado que no contaban con la formación del idioma inglés y por ende no se encontraban capacitados para impartir la enseñanza de este idioma. Por lo demás, sentían temor de no poder responder a alguna pregunta formulada por sus estudiantes si es que empleaban este recurso educativo.

El temor por el empleo del recurso It's my turn fue quedando atrás luego que el Coordinador de la Red realizara demostraciones del recurso con la participación de los mismos docentes que componían la Red. Esta experiencia hace que los docentes se muestren más favorables a probar el material con sus estudiantes, evidenciándose así un cambio sustancial en la actitud de los docentes. 
Una vez que los docentes aplicaron el recurso con sus estudiantes sus opiniones muestran un cambio, destacan lo entretenido del recurso, la buena calidad de la animación propuesta en los videos y los avances que han observado en los aprendizajes de sus estudiantes, además de la confianza que demuestran los docentes cuando abordan alguna actividad de enseñanza del idioma inglés. Ejemplo de esto último fue la participación de niños y niñas en la celebración de la English Week 2012 y 2013, realizadas en las comunas de Collipulli y Ercilla respectivamente.

\section{CONSIDERACIONES FINALES}

Los integrantes de esta Red lograron consolidarse como un equipo de trabajo, dado que sus integrantes se preocuparon por la organización para que ésta tenga éxito, poniendo su énfasis en el trabajo colaborativo, contribuyendo al logro de los objetivos propuestos por la organización, elementos que son reconocidos por Mohamed \& Mohamed (1999) como esenciales para alcanzar equipos de trabajo.

El trabajo realizado en cada una de sus reuniones mensuales y los resultados obtenidos a lo largo del tiempo hacen ver que cada miembro de la Red aportó lo mejor de sí en función de los objetivos propuestos en el plan de trabajo anual que diseñan entre todos, más allá de las limitantes personales y grupales que pudieron existir.

Otro aspecto a destacar es la sistematización de sus prácticas. En este sentido Escobar y Ramírez (2010) sostienen que el saber y conocimiento pedagógico como resultado de la sistematización de la experiencia tiene como finalidad práctica la de permitir a los sujetos la capacidad de valorar, examinar e incidir en futuro de la misma. Así, los docentes de la Red al sistematizar sus experiencias pudieron reconocer el trabajo realizado, identificar sus fortalezas y debilidades, de manera que estas últimas pudieran ser trabajadas en un futuro plan de trabajo.

En relación al acompañamiento pedagógico realizado por el Coordinador, Vezub (2011) sostiene que éste constituye una estrategia y modalidad particular para la docencia dado que promueve el desarrollo del profesorado y la innovación pedagógica a partir del análisis de las prácticas pedagógicas.

En cuanto al recurso It's my turn, la poca disposición a utilizarlo mostrado por los docentes en un inicio se entiende desde la negación al 


\section{prociênci@s v. 2, n. 2. dezembro, 2019}

cambio, el choque que produce la implantación de un nuevo recurso para el que no están preparados.

El trabajo realizado en la Red con el recurso It's my turn permitió cambiar la opinión de los docentes acerca de éste, dejando paulatinamente el temor a realizar clases de inglés por no contar con las competencias necesarias para hacerlo. Es destacable que transcurrido un tiempo de uso de este recurso educativo los docentes sientan que están en condiciones de enseñar un idioma que desconocen, valiéndose para ellos de textos, audios y videos proporcionados por el PIAP a cada uno de los establecimientos educacionales que conforman la Red.

\section{REFERENCIAS}

CRIADO, Raquel (2008), "Tesis de Doctorado Patterns of Activity Sequencing in the Teaching of English as a Foreign Language and their Effects on Learning: A Quasi-Experimental Study", Universidad de Murcia, España.

CHIARANI, Marcela, LUCERO, Margarita y PIANUCCI, Irma (2003). "Modelo de Aprendizaje Colaborativo en el ambiente $\mathrm{ACl}$ ", en:

http://sedici.unlp.edu.ar/bitstream/handle/10915/22788/Documento_compl eto.pdf?sequence $=1$ (consulta: 05 de marzo de 2019).

BIRCHAK, Barb, CONNOR, Clay, CRAWFORD, Kathleen, KAHN, Leslie, KASER, Sandy, TURNER, Susan y SHORT, Kathy. (1998), “Teacher study groups: Building community through dialogue and reflection", en: https://eric.ed.gov/?id=ED424584 (consulta: 05 de marzo de 2019).

ESCOBAR, Luís y RAMÍREZ, Jorge (2010), “La sistematización de experiencias educativas y su lugar en la formación de maestras y maestros", en:

https://aletheia.cinde.org.co/index.php/ALETHEIA/article/view/19 (consulta: 03 de marzo de 2019).

GOSDEN, Chris (1994), Social Being and Time, United Kingdom, Blackwell.

GROS, Begoña (2000), El ordenador invisible, España, Gedisa.

JOHNSON, David y JOHNSON, Frank (2000), Joining together group theory and group skills. United States, Boston Allyn and Bacon.

JOHNSON, Roger y JOHNSON, David (1986), “Action research: Cooperative learning in the science classroom", Science and Children, núm.24, pp. 3132.

MONTALTO, Donatella (1997), “Un programa para el autoaprendizaje de la lengua española en facultades de ciencias políticas", en: 
file:///C:/Users/User/Downloads/DialnetUnProgramaParaEIAutoaprendizajeDeLaLenguaEspanolaE-893276.pdf (consulta: 02 de marzo de 2019).

MUNICIPALIDAD DE COLLIPULLI (2018), “Pladeco”, en:

http://www.municipalidadcollipulli.cl/pladeco/Pladeco\%2020192022\%20Final\%20V11.pdf (consulta: 29 de octubre de 2019).

STAKE, Robert (1998), Investigación con estudio de casos, España, Ediciones Morata.

SLAVIN, Robert (1989), "Research on cooperative learning: An international perspective", Scandinavian Journal of Educational Research, vol. 33, num. 4, pp.231-243.

VEZUB, Lea (2011), “Las políticas de acompañamiento pedagógico como estrategia de desarrollo profesional docente: el caso de los programas de mentoría a docentes principiantes", en:

http://iice.institutos.filo.uba.ar/revistas (consulta: 07 de abril de 2019).

WEISS, Carol (1998), Evaluation: Methods for Studying Programs and Policies, United States, Prentice Hall.

BIBLIOTECA DEL CONGRESO NACIONAL (2015), "Reportes estadísticos comunales", en: http://reportescomunales.bcn.cl/2015/index.php/Ercilla (consulta: 22 de julio de 2017).

INSTITUTO NACIONAL DE ESTADÍSTICAS (2017), "Resultados población, sexo y edad", en: http://www.censo2017.cl/descargue-aquiresultados-de-comunas/ (consulta: 05 de mayo de 2019). 